\title{
Polymorphisms in $C A R S$ are associated with gastric cancer risk: a two-stage case-control study in the Chinese population
}

\author{
Tian Tian ${ }^{1,2} \cdot$ Ling $\mathrm{Xiao}^{2} \cdot$ Jiangbo $\mathrm{Du}^{2,3} \cdot \mathrm{Xun}_{\mathrm{Zhu}^{2}} \cdot{\text { Yayun } \mathrm{Gu}^{2}}^{2}$ \\ Na Qin ${ }^{2} \cdot$ Caiwang $\mathrm{Yan}^{2} \cdot \mathrm{Li} \mathrm{Liu}^{4} \cdot$ Hongxia $\mathrm{Ma}^{2,3} \cdot$ Yue Jiang ${ }^{2,3}$. \\ Jiaping Chen ${ }^{2,3} \cdot$ Hao $\mathrm{Yu}^{2} \cdot$ Juncheng Dai ${ }^{2,3}$
}

Received: 6 July 2016/Accepted: 16 March 2017/Published online: 13 April 2017

(c) The International Gastric Cancer Association and The Japanese Gastric Cancer Association 2017

\begin{abstract}
Background The cysteinyl transfer RNA synthetase gene (CARS) is located on chromosome band 11p15.5, which is an important tumor-suppressor gene region. Mutations in CARS have been identified in many kinds of cancers; however, evidence for a relationship between genetic variants in CARS and gastric cancer at the population level is still lacking. Thus, we explored the association of variants in CARS with gastric cancer using a two-stage casecontrol strategy in Chinese.

Methods We undertook a two-stage case-control study to investigate the association between polymorphisms in
\end{abstract}

Electronic supplementary material The online version of this article (doi:10.1007/s10120-017-0717-6) contains supplementary material, which is available to authorized users.

Tian Tian and Ling Xiao have contributed equally to this work.

$\mathrm{HaO} \mathrm{Yu}$

haoyu@njmu.edu.cn

$\triangle$ Juncheng Dai

djc@njmu.edu.cn

1 Department of Epidemiology and Biostatistics, School of Public Health, Nantong University, Nantong 226019, China

2 Department of Epidemiology and Biostatistics, Collaborative Innovation Center for Cancer Personalized Medicine, School of Public Health, Nanjing Medical University, Nanjing 211166, China

3 Jiangsu Key Lab of Cancer Biomarkers, Prevention and Treatment Collaborative Innovation Center of Cancer Medicine, Nanjing Medical University, Nanjing 211166, China

4 Digestive Endoscopy Center, The First Affiliated Hospital of Nanjing Medical University and Jiangsu Province Hospital, Nanjing, China
CARS and risk of gastric cancer with use of an Illumina Infinium ${ }^{\circledR}$ BeadChip and an ABI 7900 system.

Results Four single nucleotide polymorphisms (SNPs) were significantly associated with gastric cancer risk in both the discovery stage and the validation stage after adjustment for age and sex. In addition, the combined results of the two stages showed these SNPs were related to gastric cancer risk $\left(P_{\text {false discovery rate }} \leq 0.001\right.$ for $\quad \mathrm{rs} 384,490, \quad$ rs 729662 , rs2071101, and rs7394702). In silico analyses revealed that rs384490 and rs7394702 could affect transcription factor response elements or DNA methylation of CARS, and rs729662 was associated with the prognosis of gastric cancer. Additionally, expression quantitative trait loci analysis showed rs384490 and rs729662 might alter expression of $C A R S$-related genes.

Conclusions The potential functional SNPs in CARS might influence the biological functions of $C A R S$ or $C A R S$-related genes and ultimately modify the occurrence and development of gastric cancer in Chinese. Further large-scale population-based studies or biological functional assays are warranted to validate our findings.

Keywords Gastric cancer - Susceptibility · CARS . Polymorphism · Genetic association

\section{Introduction}

Gastric cancer is the fifth commonest malignancy worldwide, with 952,000 new cases estimated in 2012 [1]. The early detection of gastric cancer is limited, and the prognosis remains poor [2]. Although the incidence of gastric cancer and gastic cancer mortality are decreasing throughout the world, nearly two thirds of the total gastric cancer cases are in Asia, especially in China (43\% of the global gastric cancer cases) 
[3]. Besides, there are an estimated 221,478 deaths from gastric cancer each year in China, approximately half of the total gastric cancer deaths in the world [4]. In the past few decades, many risk factors have been identified, including Helicobacter pylori infection, environmental factors, and behavioral factors $[5,6]$. Previous studies revealed that these risk factors could partially explain gastric cancer development, and as only a small fraction of exposed individuals developed gastric cancer, genetic susceptibility might play an important role in the development of gastric cancer [7]. A large number of studies have been performed to explore the relationship between genetic susceptibility and gastric cancer risk, including conventional approaches (e.g., candidate gene strategy) and advanced technologies (e.g., genome-wide association study strategy) [7-9]. However, these findings are still limited to explain the genetic heterogeneity of gastric cancer, and other potential genetic evidence is needed to further explore the heritability of gastric cancer.

In 2012, a new kind of nonapoptotic cell death in unique iron-dependent form was identified, and was named ferroptosis [10]. Activation of ferroptosis could result in nonapoptotic destruction of certain cancer cells, whereas inhibition might protect organisms from neurodegeneration [10]. Hayano et al. [11] recently proposed that loss of CARS, which encodes cysteinyl transfer RNA synthetase (CARS), induces the transsulfuration pathway and affects ferroptosis, which might lead to strategies for inducing and suppressing ferroptosis in diverse contexts, including tumor contexts. CARS is a kind of aminoacyl transfer RNA synthetase; these enzymes that have long been recognized as housekeeping proteins, and growing evidence indicates that they play a key role in regulating multiple tumors [12]. The CARS gene is located on chromosome band $11 \mathrm{p} 15.5$, which is an important tumorsuppressor gene region harboring malignancy-related genes such as RRM1, CD81, and ASCL2 [13]. In many kinds of cancers (e.g., lung cancer, breast cancer, and ovarian cancer), mutations of $C A R S$ were identified, including chromosome rearrangement by the fusion between $C A R S$ and $A L K$ [14] and loss of heterozygosity of $C A R S$ by deletion [15-17]. However, evidence of a relationship between genetic variants of the CARS gene and the risk of gastric cancer at the population level is still lacking. In this study, we investigated the association of five single nucleotide polymorphisms (SNPs) in the $C A R S$ gene with gastric cancer using a two-stage case-control strategy in the Chinese population.

\section{Materials and methods}

\section{Study participants}

This study was approved by the Institutional Review Board of Nanjing Medical University, Nanjing, China. We designed a two-stage case-control study to evaluate the relationship between tagging SNPs in CARS and the risk of gastric cancer in Chinese. The recruitment of the study participants was described previously [9]. Briefly, gastric cancer patients with histopathological confirmation were consecutively recruited between 2004 and 2011 in Jiangsu province, eastern China, and patients with a history of any cancer or who had undergone radiotherapy or chemotherapy were excluded. The cancer-free controls were randomly selected from a community-based screening program for noninfectious diseases conducted in Jiangsu province. Participants were required to sign an informed consent form and complete a standard questionnaire before donating approximately $5 \mathrm{ml}$ venous blood. The controls were frequency matched to gastric cancer patients for age, sex, and geographic region. As a result, 1140 cases and 1157 controls were included in the discovery stage, and following independent replication (1140 cases and 1053 controls) was collected to validate the initial results.

\section{Selection strategy and genotyping assays of polymorphisms}

With use of the HapMap SNP database (phase II + III Feb 09, on NCBI B36 assembly, dbSNP b126), common SNPs (minor allele frequency 0.1 or greater) were screened in CARS gene regions (including the region $10 \mathrm{~kb}$ upstream of the gene) in the Han Chinese population in Beijing. With use of these criteria, 49 SNPs were selected. According to the Web-based SNP analysis tool SNPinfo Web Server (http://snpinfo.niehs. nih.gov/) [18], three potentially functional SNPs (rs384490, rs410820, and rs729662) were identified among the 49 SNPs. Linkage disequilibrium analysis with an $r^{2}$ threshold of 0.70 was further applied to filter out the tagging SNPs of the 49 SNPs (the potentially functional SNPs were included with priority). As a result, five SNPs were finally selected in this study: rs384490 $(A>C), r s 729662(A>G), r s 4758505(C>G)$, rs2071101(G $>A)$, and rs7394702( $\mathrm{A}>\mathrm{G})$.

Genotyping for the discovery stage was performed with an Illumina Infinium ${ }^{\circledR}$ BeadChip, and the genotypes were called with use of the GenTrain version 1.0 clustering algorithm in GenomeStudio V2011.1 (Illumina). A TaqMan allelic discrimination assay on the ABI 7900 system (Applied Biosystems, Foster City, CA, USA) was used for genotyping for the validation stage and SDS version 2.3 allelic discrimination software (Applied Biosystems) was used for genotype calling. Technicians were blinded to the case or control status of participants.

\section{In silico analysis}

To evaluate whether promising SNPs harbor regulatory elements, in silico analysis was performed on the basis of 
Table 1 Primary information for five single nucleotide polymorphisms in the CARS gene in the discovery stage

\begin{tabular}{|c|c|c|c|c|c|c|c|}
\hline SNP & Alleles ${ }^{\mathrm{a}}$ & Cases $(N=1140)^{\mathrm{b}}$ & Controls $(N=1547)^{\mathrm{b}}$ & $\mathrm{MAF}^{\mathrm{c}}$ & $\mathrm{HWE}^{\mathrm{d}}$ & Genotyping rate $(\%)$ & $P^{\mathrm{e}}$ \\
\hline rs 384490 & $\mathrm{~A} / \mathrm{C}$ & $748 / 356 / 33$ & $930 / 537 / 70$ & $0.186 / 0.220$ & 0.552 & 99.5 & 0.002 \\
\hline rs729662 & $\mathrm{A} / \mathrm{G}$ & $485 / 504 / 148$ & $558 / 742 / 246$ & $0.352 / 0.399$ & 1.000 & 99.9 & $<0.001$ \\
\hline rs4758505 & $\mathrm{C} / \mathrm{G}$ & $848 / 264 / 27$ & $1116 / 383 / 45$ & $0.140 / 0.153$ & 0.095 & 99.9 & 0.178 \\
\hline rs2071101 & G/A & $336 / 572 / 231$ & $541 / 743 / 262$ & $0.454 / 0.410$ & 0.793 & 99.9 & 0.001 \\
\hline rs7394702 & $\mathrm{A} / \mathrm{G}$ & $760 / 338 / 40$ & $951 / 526 / 69$ & $0.184 / 0.215$ & 0.763 & 99.9 & 0.005 \\
\hline
\end{tabular}

$H W E$ Hardy-Weinberg equilibrium, MAF minor allele frequency, SNP single nucleotide polymorphism

${ }^{a}$ Major/minor allele

b Major homozygote/heterozygote/rare homozygote between cases and controls

c MAF between cases/controls

${ }^{\mathrm{d}}$ HWE test among controls

e Derived from logistic regression analyses with an adjustment for age and sex in additive models. Values in italic are statistically significant

website resources. We analyzed the data of $\mathrm{H} 3 \mathrm{~K} 27 \mathrm{Ac}$ Mark, H3K4Me1 Mark, and H3K4Me3 Mark on seven cell lines (GM12878, H1-hESC, HSMM, HUVEC, K562, NHEK, and NHLF cells) and DNase I hypersensitivity clusters in 125 cell types from the Encyclopedia of DNA Elements (ENCODE) project through the University of California, Santa Cruz Genome Browser website (http:// genome.ucsc.edu/).

\section{Statistical analyses}

The $\chi^{2}$ test was used to evaluate distribution differences of demographic characteristics, selected variables, and genotypes between cases and controls. Deviation of the genotype distribution for each SNP from the Hardy-Weinberg equilibrium was tested by a goodness-of-fit $\chi^{2}$. Univariate and multivariate logistic regression analyses were performed to compute odds ratios (ORs) and their 95\% confidence intervals (CIs) for the associations of the genotypes with gastric cancer risk. Adjustments were made for age and sex in the logistic regression model. The Benjamini and Hochberg (false discovery rate, FDR) method was used for multiple comparison [19]. We used the $\chi^{2}$-based $Q$ test to test the heterogeneity between corresponding subgroups. For expression quantitative trait loci (eQTL) analysis, a linear regression analysis was conducted to assess the correlation between genotypes and transcript expression levels ( $\log _{10}$ transformed) of genes for the Genotype-Tissue Expression Project (GTEx) data (http://www.gtexpor tal.org/home/) [20]. The relations among positive SNPs, DNA methylation level, and expression level of the CARS gene were calculated according to publicly available data from The Cancer Genome Atlas (TCGA). Cox proportional hazards models were used to analyze the relationship between positive SNPs and prognosis of gastric cancer on the basis of TCGA data. All $P$ values presented in this study were two-sided and were considered statistically significant at $P<0.05$. All analyses were performed with $\mathrm{R}$ (version 3.1.1; R Foundation for Statistical Computing, http://www.cran.r-project.org/).

\section{Results}

The geographic characteristics for the discovery stage (1140 gastric cancer cases and 1547 controls) and the validation stage (1140 gastric cancer cases and 1053 controls) are summarized in Table S1. Briefly, no significant differences were detected for age (continuous) and sex $(P>0.05)$ between cases and controls. The genotyping call rates for the five selected SNPs were all above $99 \%$ in the discovery stage (Table 1). The observed genotypes for these SNPs were in agreement with Hardy-Weinberg equilibrium in controls for the discovery stage $(P>0.05)$. Logistic regression analyses revealed that four SNPs (rs384490, rs729662, rs2071101, and rs7394702) were significantly associated with increased risk of gastric cancer in additive models (Table 1). However, no significant association was detected between rs4758505 and gastric cancer. To further examine the associations of the four positive SNPs with gastric cancer risk, we performed stratified analyses within subgroups according to age and sex (Table S2). As a whole, the effect of variant alleles was more pronounced in males for $\operatorname{rs} 7394702$ ( $P_{\text {heterogene- }}$ ity $=0.027)$. Nevertheless, except for the previously mentioned result, no significant heterogeneity was found for ORs and their 95\% CIs between different subgroups for all four SNPs $\left(P_{\text {heterogeneity }}>0.05\right)$.

To verify the results of the discovery stage, we conducted a validation study, and we found that the four previous positive SNPs remained significantly related to gastric cancer risk in the validation stage. The call rate of genotyping was $100 \%$, and the observed genotype was in agreement with Hardy-Weinberg equilibrium in controls 
for each SNP in the validation stage (Table S3). The genotype distributions of the four SNPs between cases and controls for both the discovery stage and the validation stage are shown in Table 2. Four SNPs were significantly associated with gastric cancer risk in both the discovery stage and the validation stage (Table 2). The combined results of the two stages further showed the four SNPs were related to gastric cancer risk (Table 2, rs384490 OR 1.24, 95\% CI 1.12-1.37, $P_{\mathrm{FDR}}<0.001 ;$ rs 729662 OR $1.20,95 \%$ CI $1.11-1.31, P_{\mathrm{FDR}}<0.001$; rs $2071101 \mathrm{OR} 1.20,95 \% \mathrm{CI}$ $1.10-1.30, P_{\mathrm{FDR}}<0.001 ;$ rs 7394702 OR $1.19,95 \%$ CI $\left.1.08-1.32, P_{\mathrm{FDR}}=0.001\right)$.

Cumulative effects of the four SNPs on gastric cancer were evaluated, and the results showed a significant allele-dosage association of the number of risk alleles (or unfavorable alleles) with the risk of gastric cancer on the basis of the combined results from the two stages (Fig. 1, $P_{\text {trend }}<0.001$ ). These data indicate that those who carried more risk alleles had a higher risk of gastric cancer.

We annotated the four variants in regulatory elements cataloged on the University of California, Santa Cruz Genome Browser website (http://genome.ucsc.edu/). Visual inspection of the SNPs and histone modification peaks showed that rs384490 was situated within the enhancer element (H3K4Me1 mark and H3K27Ac histone mark) and fell into the promoter element (H3K4Me3 mark) on seven cell lines from ENCODE (Fig. 2). We found rs7394702 was situated in the enhancer element (H3K4Me1 mark and H3K27Ac histone mark) on seven cell lines from ENCODE (Fig. 2). Besides, our analysis based on TCGA data revealed that both rs7394702 and rs384490 could influence the DNA methylation levels of CARS-related CpG sites (Table S4). In addition, among these CpG sites, cg05729581 could affect the expression level of the CARS gene $(P=0.012)$. On the basis of TCGA data for gastric cancer, we also found that rs729662 was associated with the prognosis of gastric cancer after adjustment for age, sex, and pathological stage $(P=0.026)$. Therefore, we could speculate that functional SNPs located in the CARS gene may disrupt transcription factor response elements or DNA methylation levels, further affect the expression level of the CARS gene, and ultimately affect the occurrence and development of gastric cancer.

With use of GTEx (http://www.gtexportal.org/home/), a public database, eQTL analysis was performed. The results showed rs384490 was significantly associated with the expression levels of NAPILA $(P=0.021)$ and PHLDA2 $(P=0.017)$ and rs729662 was significantly associated with the expression levels of CARS-AS1 $(P=0.018)$ and SLC22A18 $(P=0.027)$ (Fig. S1).

\section{Discussion}

We investigated the association of potentially functional SNPs in the CARS gene with gastric cancer in a two-stage case-control study in the Chinese population. We found that four SNPs (384490, rs729662, rs2071101, and rs7394702) were related to increased risk of gastric cancer in both the discovery stage and the validation stage. Besides, we found that the four SNPs had cumulative effects on gastric cancer risk. In addition, further analysis showed rs384490 and rs7394702 might affect transcription factor response elements or DNA methylation of CARS, and rs384490 and rs729662 were probably eQTL SNPs for $C A R S$-related genes.

CARS is located near the imprinted gene domain on chromosome band 11p15.5, an important tumor-suppressor gene region [12]. It has been reported that alterations in this region are related to Wilms tumor, adrenocortical carcinoma, lung cancer, breast cancer, ovarian cancer, etc. [21-25]. Loss of CARS by chromosomal deletion has been observed in several cancer types, including Wilms tumor, lung cancer, embryonal rhabdomyosarcoma, and breast cancer [15-17, 26]. Hayano et al. [11] recently reported that $C A R S$ knockdown could suppress ferroptosis induced by erastin through activation of the transsulfuration pathway, which might lead to new strategies for inducing ferroptosis in specific tumor contexts, possibly including gastric cancer. Besides, Jiang et al. [27] reported that p53 inhibited cystine uptake and sensitizes cells to ferroptosis by repressing expression of SLC7A11. Moreover, the sensitivity of reactive oxygen species (ROS)-induced ferroptosis is markedly increased in p53-activated cells. Finally, Jiang et al. [27] suggested that their findings might uncover a new mode of tumor suppression based on p53 regulation of cystine metabolism, ROS responses, and ferroptosis. Thus, the change of expression of CARS and other certain important genes might affect the occurrence and development of gastric cancer by some unknown underlying mechanisms, probability including ferroptosis.

According to a Web-based analysis tool, RegulomeDB (http://www.regulomedb.org/) [28], two SNPs (rs384490 and rs7394702) are potential transcription factor binding sites (Table S5). Also, there is minimal binding evidence for rs729662 and rs2071101. Besides, two SNPs (rs384490 and rs7394702) fall into DNase I peaks, which suggests a possible mechanism for the effect of these variants on gastric cancer risk. From another online tool, SNPinfo (http://snpinfo.niehs.nih.gov/) [18], rs384490 is suggested to be potential transcription factor binding site and rs729662 may influence the exonic splicing enhancer. Variation of rs384490 may influence its interactions with transcription factors such as RNA polymerase II subunit A, 


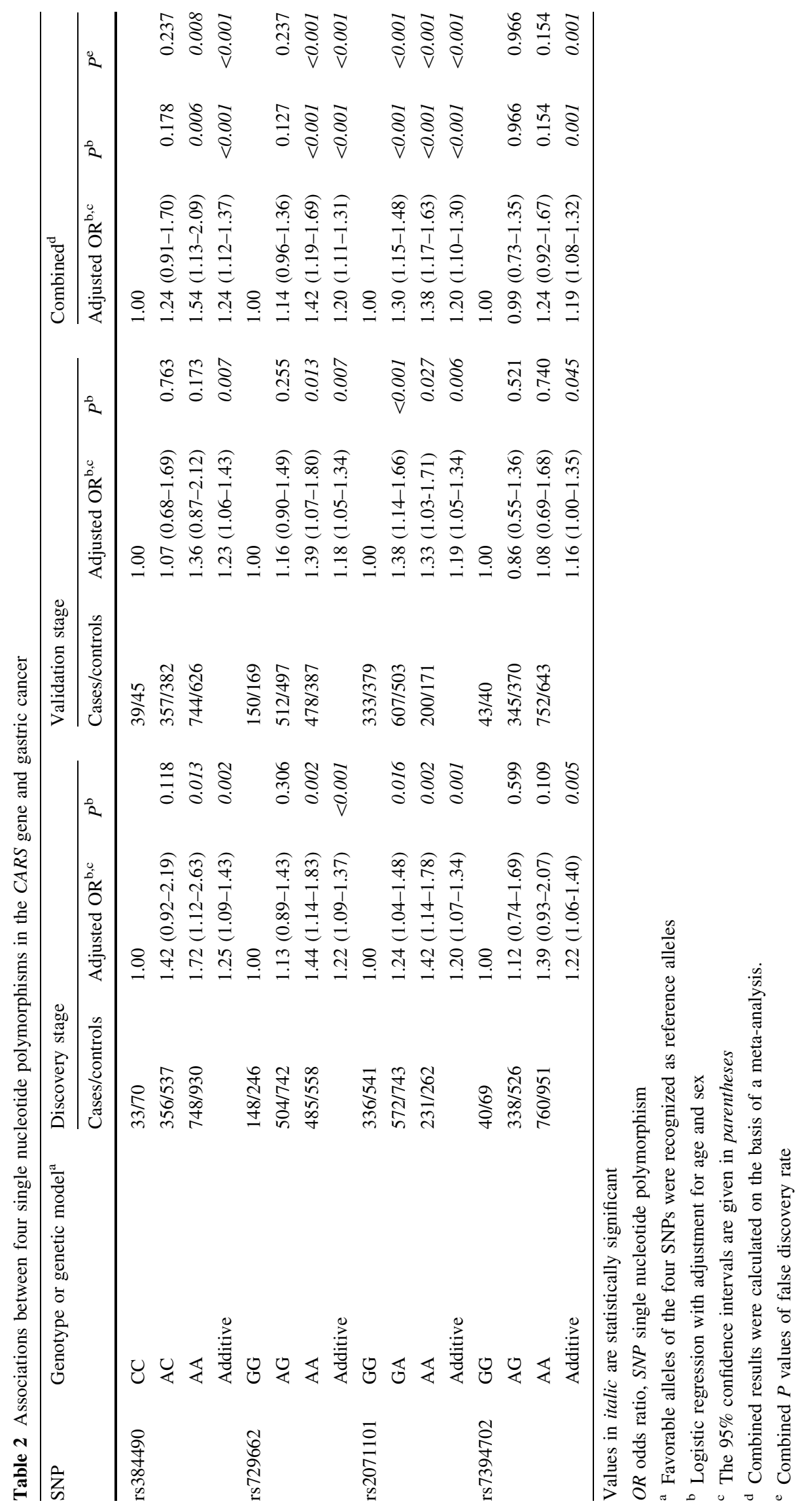




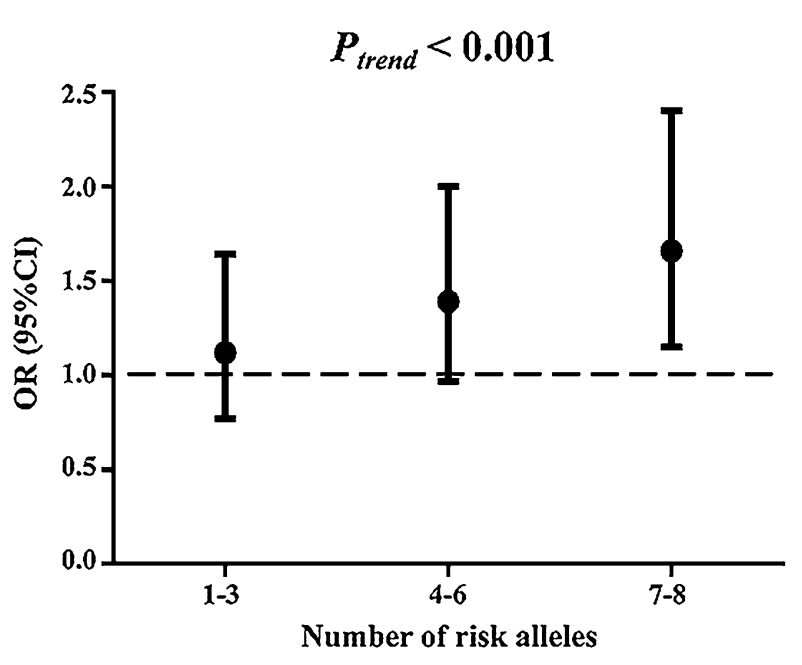

Fig. 1 Cumulative effects of rs384490, rs729662, rs2071101, and rs7394702 on gastric cancer risk. The odds ratio $(O R)$ and $95 \%$ confidence interval $(C I)$ are adjusted for age and sex on the basis of the combination of the results from the two stages. The risk alleles for the four single nucleotide polymorphisms are rs384490(A), rs729662(A), rs2071101(A), and rs7394702(A). Circles represent the ORs, and horizontal lines represent the corresponding 95\% CIs

chromodomain-helicase DNA binding protein 2, and ELK1 (as predicted by RegulomeDB) (Table S5). For example, POLR2A encodes the largest subunit of RNA polymerase II, the polymerase responsible for synthesizing messenger RNA in eukaryotes [29]. POLR2A was identified as such a gene that is almost always co-deleted with TP53 in human cancers [29, 30]. Zhou et al. [31] found that the SNP rs2071504 in the exon of the POLR2A gene would not only confer a decreased risk of gastric cancer but would also influence lymph node metastasis and the TMN stage of gastric cancer in the Chinese population. Therefore, information based on TCGA and other in silico data indicated that functional SNPs in the CARS gene might disrupt transcription factor response elements or DNA methylation and further affect the expression level of the CARS gene or other important genes.

Although direct eQTL evidence was absent for CARS itself, on the basis of related data from the GTEx database (http://www.gtexportal.org/home/) [19], rs384490 and rs729662 could influence the expression level of several other genes near the CARS gene (rs384490 is significantly associated with the expression levels of NAPIL4 and PHLDA2 and rs729662 is significantly associated with the expression levels of CARS-AS1 and SLC22A18). From statistical results, different SNPs could relate to different target genes. This situation could be explained by remote regulation among related genes, and is common in many other studies [32-34]. For example, Weinhold et al. [32] conducted eQTL analysis and found that at $7 \mathrm{p} 15.3$ the strongest association with multiple myeloma risk was for rs4487645, residing within the DNAH11 gene, which was associated with allele-specific regulation of the $C D C A 7 L$ gene (near the $D N A H 11$ gene). In our study, CARS, together with the NAP1L4, PHLDA2, SLC22A18, and CARS-AS1 genes, is located at or near the important tumor-suppressor gene region (11p15.5). CARS-AS1 is a kind of long noncoding antisense RNA that might regulate the expression of sense genes and further affect the development of

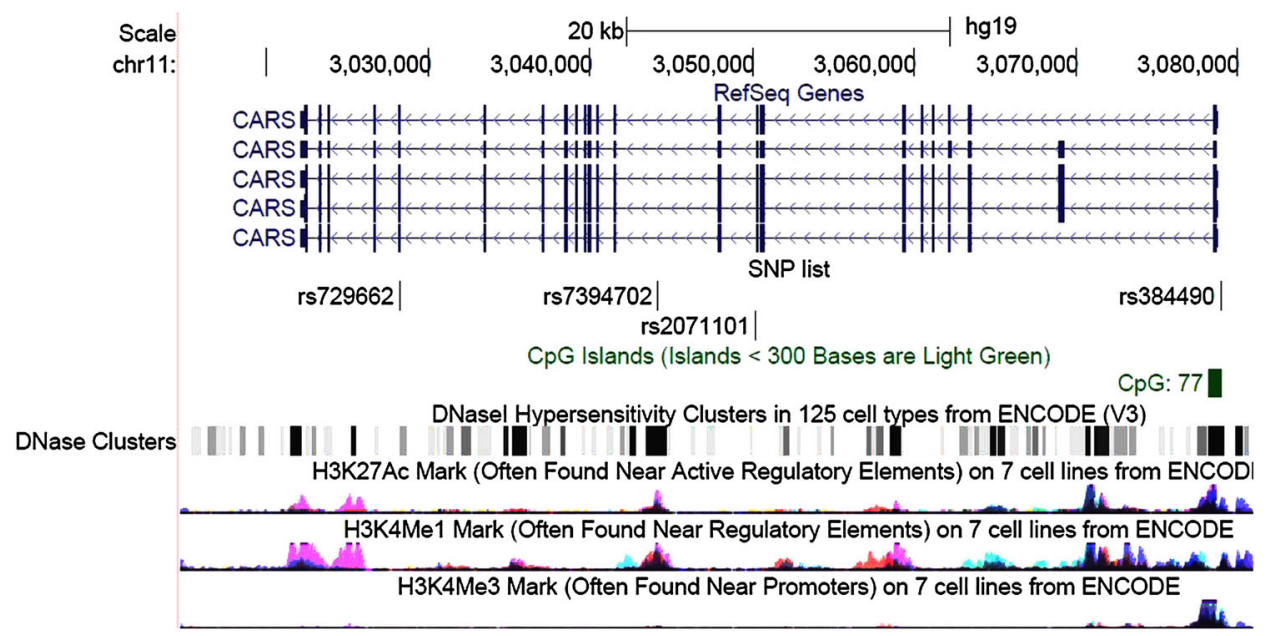

Fig. 2 Functional annotation in proximity to four promising single nucleotide polymorphisms in the CARS location. Chromatin Immunoprecipitation Sequencing (ChIP-Seq) tracks for promoter histone marks (H3K4Me3) and enhancer histone marks (H3K4Me1, H3K27Ac) in seven cell lines (GM12878, H1-hESC, HSMM, HUVEC, K562, NHEK, and NHLF cells) are present along with DNase hypersensitivity tracks from the Encyclopedia of DNA
Elements $(E N C O D E)$ data obtained through the University of California, Santa Cruz website. The black short vertical lines indicate the position of the four single nucleotide polymorphisms (rs384490, rs729662, rs2071101, and rs7394702). The light green stripe represents the $\mathrm{CpG}$ island in the region. SNP single nucleotide polymorphism 
cancers like other antisense RNAs such as FGFR3-AR1, DHRS4-AR1, and HOXA11-AS [35-37]. The cancerrelated genes PHLDA2 and SLC22A18 are similar to $C A R S$, which it is harbored in the important tumor-suppressor gene region $11 \mathrm{p} 15.5$ [38]. Besides, the NAPIL4 gene is a key candidate gene responsible for the development of cancer (including gastric cancer), and it is located near the imprinted gene domain of $11 \mathrm{p} 15.5$ mentioned before [39]. In addition, according to GeneCards (http://www.genecards.org/), CARS targets for enhancers include the genes identified by eQTL analysis mentioned earlier, which emphasizes the important relationship between these genes and CARS gene with certain potential mechanisms. Taken together, the four variants may singly or combined affect the expression of $C A R S$ or other CARS-related genes through certain potential mechanisms (e.g., influencing transcription factor response elements and DNA methylation), alter the biological function (probably including modifying ferroptosis), and ultimately affect the occurrence and development of gastric cancer. Further studies are warranted to validate the current findings.

In conclusion, this study identified four SNPs with potential function in CARS that could affect the expression of $C A R S$-related genes, and ultimately modify the occurrence and development of gastric cancer in Chinese. Further independent studies incorporating functional evaluations are warranted to confirm our findings and uncover the potentially biological mechanisms of these polymorphisms in gastric carcinogenesis.

Acknowledgements This work was funded by National Natural Science of China (81521004, 81230067, 81573238, 81422042, 81373090, 81302107), the Science Foundation for Distinguished Young Scholars of Jiangsu (BK20130042), Excellent Youth Foundation of Jiangsu (BK20160095), Jiangsu Province Clinical Science and Technology Projects (BL2012008), Key Grant of Natural Science Foundation of Jiangsu Higher Education Institutions (15KJA330002), the Priority Academic Program for the Development of Jiangsu Higher Education Institutions (Public Health and Preventive Medicine) and the Top-Notch Academic Programs Project of Jiangsu Higher Education Institution. The authors thank all study participants, research staff, and students who participated in this work.

\section{Compliance with ethical standards}

Conflict of interest The authors declare that they have no conflict of interest.

Human rights statement All procedures followed were in accordance with the ethical standards of the responsible committee on human experimentation (institutional and national) and with the Helsinki Declaration of 1964 and later versions.

Informed consent Informed consent was obtained from all patients for their being included in the study.

\section{References}

1. Colquhoun A, Arnold M, Ferlay J, Goodman KJ, Forman D, Soerjomataram I. Global patterns of cardia and non-cardia gastric cancer incidence in 2012. Gut. 2015;64:1881-8.

2. Bornschein J, Weigt J, Selgrad M, Malfertheiner P. Molecular aspects in the diagnosis of gastric cancer. Expert Opin Med Diagn. 2009;3:585-96.

3. Ferlay J, Soerjomataram I, Dikshit R, Eser S, Mathers C, Rebelo $\mathrm{M}$, et al. Cancer incidence and mortality worldwide: sources, methods and major patterns in GLOBOCAN 2012. Int J Cancer. 2015;136:E359-86.

4. Strong VE, Wu AW, Selby LV, Gonen M, Hsu M, Song KY, et al. Differences in gastric cancer survival between the U.S. and China. J Surg Oncol. 2015;112:31-7.

5. Dooley CP, Cohen H, Fitzgibbons PL, Bauer M, Appleman MD, Perez-Perez GI, et al. Prevalence of Helicobacter pylori infection and histologic gastritis in asymptomatic persons. N Engl J Med. 1989;321:1562-6.

6. Thrumurthy SG, Chaudry MA, Hochhauser D, Mughal M. The diagnosis and management of gastric cancer. BMJ. 2013;347:f6367.

7. Shi Y, Hu Z, Wu C, Dai J, Li H, Dong J, et al. A genome-wide association study identifies new susceptibility loci for non-cardia gastric cancer at $3 \mathrm{q} 13.31$ and 5p13.1. Nat Genet. 2011;43:1215-8.

8. Abnet CC, Freedman ND, Hu N, Wang Z, Yu K, Shu XO, et al. A shared susceptibility locus in PLCE1 at 10q23 for gastric adenocarcinoma and esophageal squamous cell carcinoma. Nat Genet. 2010;42:764-7.

9. Sun P, Du J, Zhu X, Ren C, Xie L, Dai N, et al. Genetic variation in the $3^{\prime}$-untranslated region of NBN gene is associated with gastric cancer risk in a Chinese population. PLoS One. 2015;10:e139059.

10. Dixon SJ, Lemberg KM, Lamprecht MR, Skouta R, Zaitsev EM, Gleason CE, et al. Ferroptosis: an iron-dependent form of nonapoptotic cell death. Cell. 2012;149:1060-72.

11. Hayano M, Yang WS, Corn CK, Pagano NC, Stockwell BR. Loss of cysteinyl-tRNA synthetase (CARS) induces the transsulfuration pathway and inhibits ferroptosis induced by cystine deprivation. Cell Death Differ. 2016;23:270-8.

12. Kim YW, Kwon C, Liu JL, Kim SH, Kim S. Cancer association study of aminoacyl-tRNA synthetase signaling network in glioblastoma. PLoS One. 2012;7:e40960.

13. Kim S, You S, Hwang D. Aminoacyl-tRNA synthetases and tumorigenesis: more than housekeeping. Nat Rev Cancer. 2011;11:708-18.

14. Debelenko LV, Arthur DC, Pack SD, Helman LJ, Schrump DS, Tsokos M. Identification of CARS-ALK fusion in primary and metastatic lesions of an inflammatory myofibroblastic tumor. Lab Invest. 2003;83:1255-65.

15. Hu RJ, Lee MP, Connors TD, Johnson LA, Burn TC, Su K, et al. A 2.5-Mb transcript map of a tumor-suppressing subchromosomal transferable fragment from $11 \mathrm{p} 15.5$, and isolation and sequence analysis of three novel genes. Genomics. 1997;46:9-17.

16. Reid LH, Davies C, Cooper PR, Crider-Miller SJ, Sait SN, Nowak NJ, et al. A 1-Mb physical map and PAC contig of the imprinted domain in $11 \mathrm{p} 15.5$ that contains TAPA1 and the BWSCR1/WT2 region. Genomics. 1997;43:366-75.

17. Zhao B, Bepler G. Transcript map and complete genomic sequence for the $310 \mathrm{~kb}$ region of minimal allele loss on chromosome segment $11 \mathrm{p} 15.5$ in non-small-cell lung cancer. Oncogene. 2001;20:8154-64. 
18. Xu Z, Taylor JA. SNPinfo: integrating GWAS and candidate gene information into functional SNP selection for genetic association studies. Nucleic Acids Res. 2009;37:W600-5.

19. Benjamini Y, Hochberg Y. Controlling the false discovery rate: a practical and powerful approach to multiple testing. J Roy Stat Soc B. 1995;57:289-300.

20. GTEx Consortium. The Genotype-Tissue Expression (GTEx) project. Nat Genet. 2013;45:580-5.

21. Reid LH, West A, Gioeli DG, Phillips KK, Kelleher KF, Araujo $\mathrm{D}$, et al. Localization of a tumor suppressor gene in $11 \mathrm{p} 15.5 \mathrm{using}$ the G401 Wilms' tumor assay. Hum Mol Genet. 1996;5:239-47.

22. Henry I, Grandjouan S, Couillin P, Barichard F, Huerre-Jeanpierre C, Glaser T, et al. Tumor-specific loss of $11 \mathrm{p} 15.5$ alleles in del11p13 Wilms tumor and in familial adrenocortical carcinoma. Proc Natl Acad Sci U S A. 1989;86:3247-51.

23. Bepler G, Garcia-Blanco MA. Three tumor-suppressor regions on chromosome $11 \mathrm{p}$ identified by high-resolution deletion mapping in human non-small-cell lung cancer. Proc Natl Acad Sci U S A. 1994;91:5513-7.

24. Gudmundsson J, Barkardottir RB, Eiriksdottir G, Baldursson T, Arason A, Egilsson V, et al. Loss of heterozygosity at chromosome 11 in breast cancer: association of prognostic factors with genetic alterations. Br J Cancer. 1995;72:696-701.

25. Gabra H, Watson J, Eccles D, Taylor L, Taylor K, Cohen B, et al. A statistical analysis of chromosome 11 and 17 loss of heterozygosity in epithelial ovarian cancer. Int $\mathrm{J}$ Oncol. 1996;8:625-31.

26. Xu XL, Wu LC, Du F, Davis A, Peyton M, Tomizawa Y, et al. Inactivation of human SRBC, located within the 11p15.5-p15.4 tumor suppressor region, in breast and lung cancers. Cancer Res. 2001;61:7943-9.

27. Jiang L, Kon N, Li T, Wang SJ, Su T, Hibshoosh H, et al. Ferroptosis as a p53-mediated activity during tumour suppression. Nature. 2015;520:57-62.

28. Boyle AP, Hong EL, Hariharan M, Cheng Y, Schaub MA, Kasowski M, et al. Annotation of functional variation in personal genomes using RegulomeDB. Genome Res. 2012;22:1790-7.

29. Liu Y, Zhang X, Han C, Wan G, Huang X, Ivan C, et al. TP53 loss creates therapeutic vulnerability in colorectal cancer. Nature. 2015;520:697-701.
30. Errico A. Colorectal cancer: POLR2A deletion with TP53 opens a window of opportunity for therapy. Nat Rev Clin Oncol. 2015;12:374.

31. Zhou Y, Du WD, Chen G, Ruan J, Xu S, Zhou FS, et al. Association analysis of genetic variants in microRNA networks and gastric cancer risk in a Chinese Han population. J Cancer Res Clin Oncol. 2012;138:939-45.

32. Weinhold N, Meissner T, Johnson DC, Seckinger A, Moreaux J, Försti A, et al. The 7p15.3 (rs4487645) association for multiple myeloma shows strong allele-specific regulation of the MYCinteracting gene CDCA7L in malignant plasma cells. Haematologica. 2015;100:e110-3.

33. Ke J, Lou J, Chen X, Li J, Liu C, Gong Y, et al. Identification of a functional variant for colorectal cancer risk mapping to chromosome 5q31.1. Oncotarget. 2016;7:35199-207.

34. Closa A, Cordero D, Sanz-Pamplona R, Solé X, Crous-Bou M, Paré-Brunet $\mathrm{L}$, et al. Identification of candidate susceptibility genes for colorectal cancer through eQTL analysis. Carcinogenesis. 2014;35:2039-46.

35. Sun J, Wang X, Fu C, Wang X, Zou J, Hua H, et al. Long noncoding RNA FGFR3-AS1 promotes osteosarcoma growth through regulating its natural antisense transcript FGFR3. Mol Biol Rep. 2016;43:427-36.

36. Yang Y, Su Z, Song X, Liang B, Zeng F, Chang X, et al. Enhancer RNA-driven looping enhances the transcription of the long noncoding RNA DHRS4-AS1, a controller of the DHRS4 gene cluster. Sci Rep. 2016;6:20961.

37. Wang Q, Zhang J, Liu Y, Zhang W, Zhou J, Duan R, et al. A novel cell cycle-associated lncRNA, HOXA11-AS, is transcribed from the 5-prime end of the HOXA transcript and is a biomarker of progression in glioma. Cancer Lett. 2016;373:251-9.

38. Altinoz MA, Elmaci I, Ince B, Ozpinar A, Sav AM. Hemorphins, and $11 \mathrm{p} 15.5$ chromosomal region in cancer biology and immunity with special emphasis for brain tumors. J Neurol Surg A Cent Eur Neurosurg. 2016;77:247-57.

39. Li S, Li J, Tian J, Dong R, Wei J, Qiu X, et al. Characterization, tissue expression, and imprinting analysis of the porcine CDKN1C and NAP1L4 genes. J Biomed Biotechnol. 2012;2012:946527. 